\title{
Maternal Body Mass Index and Ethnicity in Relation to The Adverse Outcomes of Large for Gestational Age and Gestational Diabetes Mellitus in a Retrospective Cohort of Australian Pregnant Women.
}

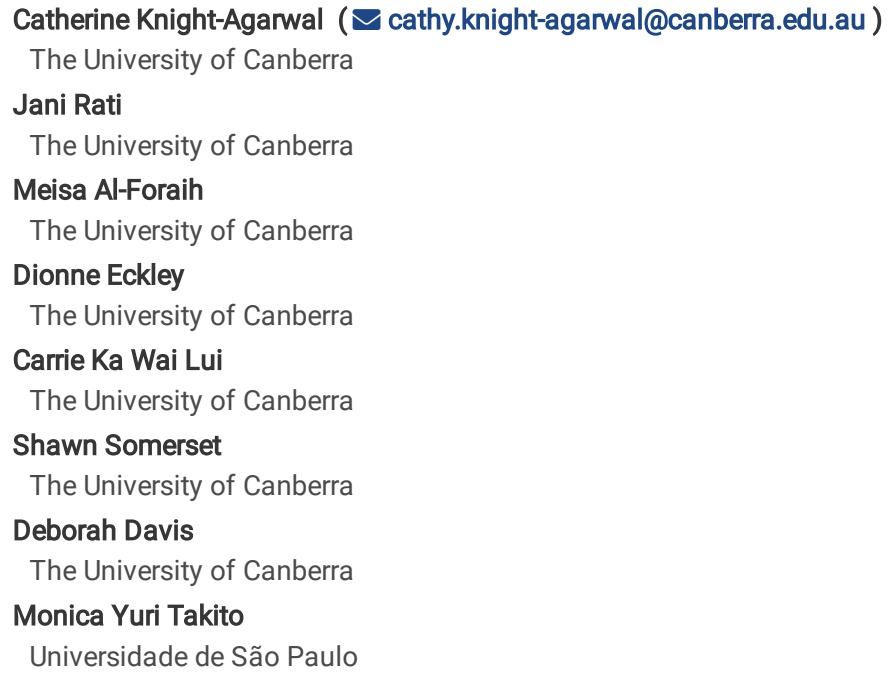




\section{Abstract}

Background: The prevalence of maternal overweight and obesity has been increasing. This research explored the association between maternal body mass index and ethnicity in relation to the adverse outcomes of large for gestational age and gestational diabetes mellitus.

Method: $A$ retrospective cohort study was undertaken with 27814 Australian women of various ethnicities, who gave birth to a singleton infant between 2008 and 2017. Variables were examined using logistic regression.

Results: A significantly higher proportion of large for gestational age infants were born to overweight and obese women compared to those who were classified as underweight and healthy weight. Asian-born women with a body mass index of $\geq 40 \mathrm{~kg} / \mathrm{m}^{2}$ had an adjusted odds ratio of 9.926 ( 3.859 - 25.535 ) for birthing a large for gestational age infant whereas Australian-born women had an adjusted odds ratio of 2.661 (2.256 - 3.139 ) for the same outcome. Women born in Australia were at high risk of birthing a large for gestational age infant in the presence of insulin controlled gestational diabetes mellitus, but this risk was not significant for those with the diet-controlled type. Asian-born women did not present an elevated risk of birthing a large for gestational infant, in either the diet controlled, or insulin controlled gestational diabetes mellitus groups.

Conclusion: Large for gestational age and gestational diabetes mellitus are adverse pregnancy outcomes that can lead to significant maternal and neonatal morbidity. Women who are overweight or obese, and considering a pregnancy, are encouraged to seek culturally appropriate nutrition and weight management advice during the periconception period.

\section{Background}

In Australia, the prevalence of maternal overweight and obesity has been increasing largely in line with prevalence in the general population [1, 2] and indeed other industrialised countries. [3, 4]. Data from the National Health Survey reported that there were over 2.5 million overweight and obese women residing in Australia between 2014-2015 (approximately $29 \%$ of all females aged 18 years and over) [1, 2]. Comparable figures from the nation's capital of Canberra have been reported [1].

Numerous studies have shown that maternal pre-pregnancy BMI of $\geq 30 \mathrm{~kg} / \mathrm{m}^{2} \mathrm{~kg} / \mathrm{m}^{2}$ increases the prevalence of pregnancy complications, such as GDM and LGA, which can in turn elevate offspring risk for chronic disease in later life[5-7]. In addition, evidence exists that being born LGA is a predictor of obesity in adulthood [11]. The proportion of newborns with macrosomia, defined as having a birth weight of over 4 kilograms, ranges from less than $1.0-14.9 \%$ in developing countries to as high as $20 \%$ in northern Europe [12-13]. An Australian retrospective cohort study including 24,161singleton births between 20092015 demonstrated a significant association between overweight and obesity in early pregnancy and increased risks of preterm birth (PTB), LGA and admissions to neonatal intensive care units (NICU) [8]. Similar outcomes have been reported in North America, Asia, Europe and the Middle East [9-23]. Several studies have also reported a link between maternal obesity and insulin resistance in adult offspring as well as higher rates of type 2 diabetes mellitus and impaired adult cardiovascular health $[12,24,25]$. Such relationships have also been found in Australian cohorts, especially amongst women from Asian backgrounds, with those entering pregnancy with an elevated pre-pregnancy BMI at even greater risk [9-10, 26].

A high proportion of the Australian population was born in countries other than Australia[2]. In 2019, there were over 7.5 million migrants living in Australia which is just under $30 \%$ of the total population. England continues to be the largest group of overseas-born individuals residing in Australia however second and third place are China and India, respectively. Median age of Chinese and Indian women migrating to Australia is 34 years with the vast majority being childless but of child-bearing age [1].

Previous studies have shown that migrants often have difficulty acculturating to their new country, which has consequences for dietary practices and other determinants of well-being $[27,28]$. The increased risk of chronic disease manifested in migrants from low middle income countries (LMICs) is related in part to the transition from food environments of undernutrition, to overnutrition[29].

Given the high proportion of Australian women born elsewhere and their equally high rates of overweight and obesity[30], they may be particularly vulnerable to the consequences of excessive body weight. This study, a secondary analysis of routinely collected clinical data, was undertaken to determine comparative risk of both Australian-born and Asian-born women regarding BMI, LGA and GDM. Such data is vital for informing the development of targeted interventions to maximize positive birthing outcomes particularly in women with high BMI's and from various ethnicities.

\section{Methods}

This retrospective cohort study was conducted using data from the Birthing Outcomes System (BOS) at a major tertiary institution in eastern Australia between the 1st of January 2008 and the 31 st of December 2017. There were 30,121 births over this period, with the hospital being the major maternity centre for a catchment population of 550,000. However, stillbirths and multiple pregnancies were excluded as were births where maternal BMI had not been recorded. This left 27,814 birth events for analysis. Ethical approval was obtained from the relevant Health Research Ethics and Governance Office. (no: ETHLR.18.048).

\section{Data Assessment}

Maternal BMI was derived from measured height $(\mathrm{cm})$ and weight $(\mathrm{kg})$ recorded at the first antenatal appointment (usually at 12-14 weeks gestation) [26]. Classification of BMI was defined, according to WHO cut-offs, into four groups: underweight $\left(\leq 18.5 \mathrm{~kg} / \mathrm{m}^{2}\right)$; healthy weight $\left(18.5-24.9 \mathrm{~kg} / \mathrm{m}^{2}\right)$; overweight $(25$ $\left.29.9 \mathrm{~kg} / \mathrm{m}^{2}\right)$; obese class I $\left(30-34.9 \mathrm{~kg} / \mathrm{m}^{2}\right)$; obese class II $\left(35-39.9 \mathrm{~kg} / \mathrm{m}^{2}\right)$ and obese class III $\left(>40 \mathrm{~kg} / \mathrm{m}^{2}\right)[2]$. 
Other demographic information which was collected included maternal age, maternal country of birth, relationship status, employment, smoking (both maternal and paternal), parity, and obstetric outcomes such as GDM, hypertensive disorders of pregnancy and premature rupture of membranes.

Maternal place of birth is recorded in the BOS database. Women were categorised into three broad groups: 'all'(regardless of ethnicity) 'Australian-born' and 'Asian-born'. The Standard Australian Classification of Countries (SACC), Second Edition [23] was used to define the nations to be included in this final category (for example China, India, Pakistan).

Gestational age was calculated from either the last menstrual period or the earliest ultrasound examination. The Australian national birthweight percentiles published by Dobbins et al., were used to calculate LGA defined as a birthweight $>90$ th percentile for gestational age [26, 31, 32]. Birthweight results were expressed as SD ( $z)$ scores corrected for gestation at time of birth.

Maternity complications such as GDM and hypertensive disorders of pregnancy were defined according to the World Health Organisations (WHO's) International Statistical Classification of Diseases and Related Health Problems manual [25]. Screening for GDM is universally conducted at the study hospital between 24- and 28-weeks' gestation with a $75 \mathrm{~g}$ oral glucose tolerance test (OGTT). A positive diagnosis is made if the fasting plasma glucose is 5.1 - 6.9 $\mathrm{mmol} / \mathrm{L}$ or if the 2-hour post glucose load is $8.5-11.0 \mathrm{mmol} / \mathrm{L}$. Women with GDM receive group education from experienced dietitians and diabetes educators. This includes blood glucose monitoring, carbohydrate counting and recommended physical activity levels. Women are strongly encouraged to attend individual follow-up appointments either weekly or fortnightly in line with the Australasian Diabetes in Pregnancy Society (ADIPS) consensus guidelines for the testing and diagnosis of hyperglycaemia in pregnancy [26]. Data are entered into the database by clinicians contemporaneously or as soon as practicable after an episode of care with regular validation checks by the system administrator. Mandatory reporting fields are validated by the Epidemiology Section of the Department of Population Health at the jurisdiction level.

\section{Statistical Analysis}

Descriptive analysis was reported using means and standard deviations for continuous variables and frequencies and percentages for categorical variables. Binary logistic regression was performed, to assess the relationship between maternal BMI, GDM and LGA. Following this, multivariate binary logistic regression, using the forced entry method, was applied to associations found to be significant at the bivariate level. All models were adjusted for parity, baby gender, marital status, smoking, maternal country of birth, employment and premature rupture of membranes. These covariates are considered by clinicians working in the filed as important and have been used in similar published analyses on this topic $[8,26,33,34]$.

Cook's distance values were used to examine for multivariate outliers and influential data points. All cases included in the study had Cook's D values below one. No signs of multicollinearity were observed, and an acceptable goodness of fit model was found. Statistical significance was set at $\mathrm{p}<0.05$. Analyses were conducted using SPSS version 24 (SPSS Inc., Chicago, USA) [27].

\section{Results}

A total of 27, 814 singleton birth events with accompanying maternal BMls, were included in the study (Table 1). Of these women: 1,544 (5.6\%) were underweight; 13,948 (50.0\%) had normal BMls; 6,832 (24.6\%) were overweight; 2,967 (10.7\%) were obese l; 1,412 (5.1\%) were obese Il; and 1,111 (4.0\%) were obese III. Neonatal outcomes included: 292 (1.0\%) extremely low birthweight (<1,000g); 235 (0.8\%) very low birthweight (1000-1,499-g); 1,528 (5.5\%) low birthweight (1,500-2,499g); 22,541 (81.0\%) normal (2,500-3,999); 2,710 (9.7\%) large (4,000-4,499g); and 508 (1.8\%) exceptionally large (4,500g and more). For gestational age there was: 265 (1.0\%) of extreme prematurity (<28 weeks); 2,045 (7.4\%) preterm (28-36.9 weeks); 25,248 (90.8\%) born at term (37-41.9 weeks); and $256(0.9 \%)$ born post-term (42 weeks and more).

Table 1. Frequency, percentage and unadjusted odds ratio's for neonatal outcomes according to maternal and peripartum characteristics in a cohort of pregnant Canberran women, 2008-2017 


\begin{tabular}{|c|c|c|c|}
\hline & \multirow{2}{*}{$\begin{array}{c}\text { Total } \\
\mathbf{N}\end{array}$} & \multicolumn{2}{|c|}{ Large for gestational AGE } \\
\hline & & n (\%) & OR crude $\left(\mathrm{Cl}_{95 \%}\right)$ \\
\hline \multicolumn{4}{|c|}{ BMI $\left(\mathrm{kg} / \mathrm{m}^{2}\right)$} \\
\hline$<18.5$ & 1544 & $78(5.1)$ & $0.559(0.442 ; 0.707)$ \\
\hline $18.5-24.9$ & 13948 & $1213(8.7)$ & 1 \\
\hline $25-29.9$ & 6832 & $955(14.0)$ & $1.706(1.559 ; 1.867)$ \\
\hline $30-34.9$ & 2967 & $534(18.0)$ & $2.304(2.063 ; 2.574)$ \\
\hline $35-39.9$ & 1412 & $293(20.8)$ & $2.749(2.386 ; 3.167)$ \\
\hline$\geq 40.0$ & 1111 & $279(25.1)$ & $3.521(3.037 ; 4.082)$ \\
\hline \multicolumn{4}{|c|}{ Baby gender } \\
\hline Female & 13387 & $1517(11.3)$ & 1 \\
\hline Male & 14414 & $1835(12.7)$ & $1.141(1.062 ; 1.227)$ \\
\hline \multicolumn{4}{|l|}{ Parity } \\
\hline 0 & 12256 & $1055(8.6)$ & 1 \\
\hline 1 & 9602 & $1316(13.7)$ & $1.686(1.548 ; 1.837)$ \\
\hline 2 & 3810 & $621(16.3)$ & $2.067(1.858 ; 2.330)$ \\
\hline 3 & 1324 & 209 (15.8) & $1.990(1.695 ; 2.337)$ \\
\hline$\geq 4$ & 820 & $150(18.3)$ & $2.377(1.970 ; 2.868)$ \\
\hline \multicolumn{4}{|c|}{ Mother work } \\
\hline No & 15253 & $1854(12.2)$ & 1 \\
\hline Yes & 12561 & $1498(11.9)$ & $0.979(0.910 ; 1.052)$ \\
\hline \multicolumn{4}{|c|}{ Country of birth } \\
\hline Australian & 19329 & $2659(13.8)$ & 1 \\
\hline Asian & 5054 & $301(6.0)$ & $0.397(0.351 ; 0.449)$ \\
\hline Other & 3394 & 391 (11.5) & $0.816(0.729 ; 0.914)$ \\
\hline \multicolumn{4}{|c|}{ Women smokes during pregnancy } \\
\hline \multicolumn{4}{|l|}{ No } \\
\hline \multicolumn{4}{|l|}{ Yes } \\
\hline & 25251 & $3139(12.4)$ & 1 \\
\hline & 2368 & $197(8.3)$ & $0.639(0.550 ; 0.743)$ \\
\hline \multicolumn{4}{|c|}{ Partner smokes } \\
\hline No & 23916 & $2941(12.3)$ & 1 \\
\hline Yes & 3898 & $411(10.5)$ & $0.841(0.754 ; 0.938)$ \\
\hline \multicolumn{4}{|c|}{ Married or with a partner } \\
\hline \multicolumn{4}{|l|}{ No } \\
\hline \multirow[t]{2}{*}{ Yes } & 3261 & $366(11.2)$ & 1 \\
\hline & 24553 & $2986(12.2)$ & $1.095(0.976 ; 1.229)$ \\
\hline \multicolumn{4}{|c|}{ Premature rupture of membrane } \\
\hline \multicolumn{4}{|l|}{ No } \\
\hline \multicolumn{4}{|l|}{ Yes } \\
\hline & 27217 & $3297(12.1)$ & 1 \\
\hline
\end{tabular}




\begin{tabular}{|c|c|c|c|}
\hline & 597 & $55(9.2)$ & $0.736(0.557 ; 0.974)$ \\
\hline \multicolumn{4}{|l|}{ PIHD } \\
\hline No & 26395 & 3138 (11.9) & 1 \\
\hline Yes & 1419 & $214(15.1)$ & $1.316(1.133 ; 1.529)$ \\
\hline \multicolumn{4}{|c|}{ Gestational } \\
\hline \multicolumn{4}{|l|}{ Diabetes } \\
\hline \multicolumn{4}{|l|}{ (GDM) } \\
\hline No & 24793 & $2849(11.5)$ & 1 \\
\hline Yes & 3021 & $503(16.7)$ & $1.380(1.239 ; 1.537)$ \\
\hline
\end{tabular}

BMI: Body Mass Index, PIHD: Pregnancy-induced hypertension. Significant values are bolded.

Table 2. Multivariate analysis of the association between LGA, GDM and maternal BMI. 


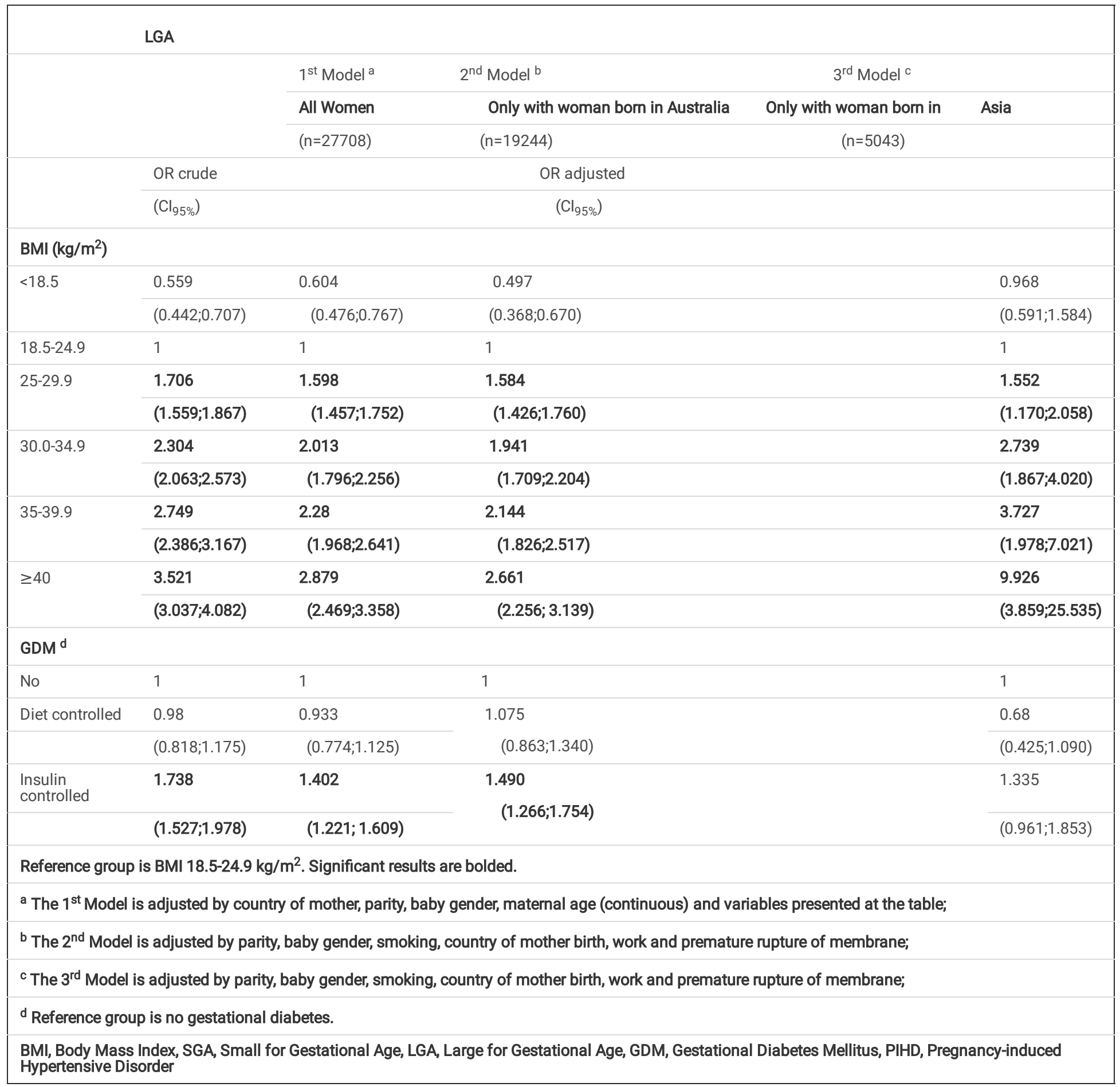

There was no significant difference observed between underweight and healthy weight women in terms of the proportion of LGA babies. However, there was a significantly higher proportion of LGA neonates born to overweight and obese women compared to underweight and healthy weight women (Bonferroni, $\mathrm{p}$ <0.001). As maternal BMI increased so did LGA. This trend was observed in all models which included the whole cohort plus Australian-born and Asian-born cohorts. Australian-born women with a BMI of $\geq 40 \mathrm{~kg} / \mathrm{m}^{2}$ had an AOR of 2.661 (Cl: 2.256; 3.139) for birthing an LGA infant. Furthermore, women born in Asia with a BMI of $\geq 40 \mathrm{~kg} / \mathrm{m}^{2}$ were found to have an AOR of 9.926 (Cl: $3.859 ; 25.535$ ) for birthing an LGA infant.

Finally, the effect of diabetes treatment (diet controlled vs. insulin controlled) during pregnancy was found to be statistically significant for LGA depending on the woman's country of birth (Table 2). Women born in Australia showed higher risk for LGA in the presence of insulin controlled GDM, however no significant risk for LGA was found in diet controlled GDM. Asian-born women did not demonstrate significant risk for LGA in either the diet controlled or insulin controlled GDM models.

\section{Discussion}


In our study both Australian and Asian-born women with a high BMI had a significantly elevated risk of birthing an LGA infant when compared to their healthy weight counterparts. Our results show that Asian-born women with a BMI of $\geq 40 \mathrm{~kg} / \mathrm{m}^{2}$ were almost 10 times more likely to birth an LGA infant compared to Asian-born women within a healthy weight range despite adjusting for covariates such as parity and maternal age. A recent US population-based cohort study of 2,842,278 singleton births reported the odds of having an LGA baby was greatest for obese Asian Americans with an AOR of 2.05 (Cl:1.91, 2.20) compared to other racial/ethnic groups in the same class of BMI [28].

In our study, Australian-born women with insulin controlled GDM were significantly more likely to have an LGA baby when compared to women with either diet controlled GDM or no GDM. A recent retrospective study undertaken with an Australian maternity cohort $(n=73,517)$ reported that Chinese-born women had a 4-fold higher risk of GDM, despite having a lower pre-pregnancy BMI, than Caucasian women. Interestingly, after adjusting for confounders, Chinese-born women with GDM had a lower risk of birthing an LGA infant compared to their Caucasian counterparts. The authors suggested this may be related to physiological differences, migration patterns, and GDM experiences in a sociocultural context [29]. A Canadian retrospective cohort study was undertaken to determine the association between Chinese or South Asian (Indian) ethnicity and adverse neonatal and maternal outcomes for women with GDM compared to the general population. In contrast to infants of women from the general population (55.5\%), infants of Chinese mothers had a lower risk of an adverse outcome at birth (42.9\%, AOR 0.63, Cl: 0.58-0.68), whereas infants of South Asian (Indian) mothers had a higher risk (58.9\%, AOR 1.15, Cl: 1.07-1.23). Clearly, the likelihood of GDM complications differed significantly between Chinese-born and Indian-born women and the general population [30]. Despite observing in our cohort an upward trend for LGA in Asian-born women with insulin dependent GDM this was not statistically significant.

Another retrospective cohort study conducted by Yang and colleagues revealed that two separate variables, namely pre-pregnancy overweight and GDM, were both associated with an increased risk of LGA and that they also displayed a synergistic effect on its occurrence. In their analysis, they adjusted for treatment modes such as insulin therapy or diet-control only, in order to minimize possible confounding effects which could result from different types of GDM management. The authors found co-existence of high BMI and GDM predisposed women to a 5-fold risk of LGA when compared with women who had a BMI within the healthy range with no GDM [31-32].

Pedersen's hypothesis states that elevated levels of maternal blood glucose can transverse the placenta but insulin cannot [33]. As a result, when additional glucose is delivered across the placenta the fetuses' pancreatic islet cells are stimulated to secrete insulin for glucose uptake, leading to fetal hyperinsulinemia and hyperglycemia. A consequence of this process is excessive accumulation of fetal adipose tissue leading to an increase in body weight. In addition, it is widely recognised that high maternal BMI can aggravate offspring obesity through genetic predisposition and the in-utero environment [11]. Such metabolic derangements could be a plausible explanation as to why, within our cohort, both Australian-born and Asian-born women with diet-controlled GDM did not display a higher risk of LGA when compared to their non-GDM counterparts. Mild hyperglycaemia is likely to be controlled by dietary measures, especially in women who are highly motivated. In fact, those with diet-controlled GDM in our cohort appear to be at lower risk of LGA compared to those with either insulincontrolled GDM or no GDM. However, this trend was not statistically significant.

There are limitations to our study that should be acknowledged. Use of a single Asian-born group may not provide an accurate representation of the outcomes reported for LGA and GDM. There may be considerable heterogeneity, among Asian subgroups, highlighting the importance of disaggregation to assess ethnic differences. We did not have access to information on the gestational week when OGTT was performed, the degree of glycaemic control achieved by women or ethnic differences in adherence with GDM treatment. Detailed information on nutrition, physical activity, and gestational weight gain (GWG), which may be factors underlying the racial differences in LGA risk, were unavailable. In addition, ethnicity-specific cut-off points are not routinely used at the study hospital for the calculation of maternal BMI. Nevertheless, ethnicity was controlled for in our analyses. We used birthweight percentiles for all singleton infants born in Australia between 1998 and 2007 to calculate LGA [24]. It would be interesting to see if our results differed using birth weight percentiles for each of the racial groups identified in our cohort. Finally, we know that obesity increases risk of GDM and GDM increases risk of LGA. However, we were unable to confirm the inter-relationship between these two variables for increased risk of LGA. Future analysis (using this dataset) could involve mediation analysis between all three variables.

While Asian-born women residing in Australia are eligible to access the same health services as women born in Australia, they may experience several challenges including connecting with antenatal care services, insufficient support, English language difficulties and transport issues. From a clinical perspective, the critical role that both maternal BMI and ethnicity play in their association with LGA and GDM risk is important to elucidate so that culturally appropriate interventions may be developed for application before and during pregnancy.

\section{Conclusion}

Because high maternal BMI often precedes GDM, decreasing the prevalence of overweight and obesity could reduce the prevalence of both GDM and LGA. However, to increase the percentage of women entering pregnancy with a healthy BMI, greater public health strategies are needed. Furthermore, women of reproductive age who are contemplating pregnancy should be encouraged by their primary health care providers to seek nutrition and weight management advice when indicated.

\section{Abbreviations}

ACT - Australian Capital Territory, BOS- Birthing Outcomes Systems, BMI -Body Mass Index, LGA- Large for Gestational Age, GDM-Gestational Diabetes Mellitus, PIHD- Pregnancy-induced Hypertensive Disorder, HBW- High Birth Weight, HIC- High- income Countries, OGTT - Oral Glucose Tolerance Test, GWG Gestational Weight Gain.

\section{Declarations}


Ethics approval and consent to participate

The authors declare that all experiments on human subjects were conducted in accordance with the Declaration of Helsinki. Written consent of study participants was waivered by the ACT Health Human Research Ethics Committee (HREC) as the retrospective data collected from BOS was already deidentified. The authors also certify that formal approval to conduct the research described has been obtained from the ACT HREC (ETHLR.18.048).

\section{Consent for publication}

Not applicable

\section{Availability of data and materials}

The datasets used and/or analysed during the current study are available from the corresponding author on reasonable request.

\section{Competing interests}

There are no conflicts of interest

\section{Funding}

We would like to thank the University of Sao Paulo, Brazil for their support (FAPESP GRANT - 2017/19280-4).

\section{Authors' contributions}

CKA: Conceptualization, Methodology, Data curation; Supervision, Visualization, Writing-Original draft preparation, Writing-Reviewing and Editing; MYT: Funding acquisition, Methodology, Formal analysis, Supervision, Writing-Reviewing and Editing; RJ: Data curation, Supervision, Writing-Reviewing and Editing, Visualization, Supervision; MA: Data curation, Writing-Reviewing and Editing, Visualization; DA: Methodology, Writing- Reviewing and Editing; CL: Methodology, Writing-Reviewing and Editing; SS: Supervision, Writing- Reviewing and Editing; DD: Supervision, Writing- Reviewing and Editing.

\section{Acknowledgements}

We would like to thank Dr. David Knight FRANZCOG FRACGP for his help with data collection.

\section{References}

1. AlHW. A picture of overweight and obesity in Australia. 2017; Cat. no. PHE 216:[Available from: https://www.aihw.gov.au/getmedia/172fba28-785e-4a08ab37-2da3bbae40b8/aihw-phe-216.pdf.aspx?inline=true.

2. Australian Bureau of Statistics. National Health Survey. 2015; Catalogue No. 4364.0.55.001:[Available from: https://www.ausstats.abs.gov.au/Ausstats/subscriber.nsf/0/CDA852A349B4CEE6CA257F150009FC53/\$File/national\%20health\%20survey\%20first\%20rt 15.pdf.

3. Williams, E.P., et al., Overweight and Obesity: Prevalence, Consequences, and Causes of a Growing Public Health Problem. Current Obesity Reports, 2015. 4(3): p. 363-370.

4. Chen, C., X. Xu, and Y. Yan, Estimated global overweight and obesity burden in pregnant women based on panel data model. PloS one, 2018. 13(8): p. e0202183-e0202183.

5. World Health Organization, Waist Circumference and Waist-Hip Ratio. Report of the WHO Expert Consultation; Geneva, 8-11 December 2008, 2011.

6. Aune, D., et al., Maternal body mass index and the risk of fetal death, stillbirth, and infant death: A systematic review and meta-analysis. JAMA, 2014. 311(15): p. 1536-1546.

7. Reynolds, R. and L. Stirrat, Effects of maternal obesity on early and long-term outcomes for offspring. Research and Reports in Neonatology, 2014. 2014: p. 43.

8. Yang, Z., et al., Contribution of maternal overweight and obesity to the occurrence of adverse pregnancy outcomes. Australian and New Zealand Journal of Obstetrics and Gynaecology, 2019. 59(3): p. 367-374.

9. Cedergren, M.I., Maternal morbid obesity and the risk of adverse pregnancy outcome. Obstetrics \& Gynecology, 2004. 103(2): p. 219-224.

10. Driul, L., et al., Prepregnancy body mass index and adverse pregnancy outcomes. Arch Gynecol Obstet, 2008. 278(1): p. 23-6.

11. Poston, L., et al., Preconceptional and maternal obesity: epidemiology and health consequences. The Lancet Diabetes \& Endocrinology, 2016. 4(12): p. 1025-1036.

12. Valsamakis, G., et al., Effect of maternal obesity on pregnancy outcomes and long-term metabolic consequences. Hormones (Athens), 2015. 14(3): p. 34557.

13. O'Reilly, J.R. and R.M. Reynolds, The risk of maternal obesity to the long-term health of the offspring. Clinical Endocrinology, 2013. 78(1): p. 9-16.

14. Shaukat, S. and U. Nur, Effect of prepregnancy maternal BMI on adverse pregnancy and neonatal outcomes: results from a retrospective cohort study of a multiethnic population in Qatar. BMJ Open, 2019. 9(9): p. e029757.

15. Ratnasiri, A.W.G., et al., Trends in maternal prepregnancy body mass index (BMI) and its association with birth and maternal outcomes in California, 20072016: A retrospective cohort study. Plos one, 2019. 14(9): p. e0222458-e222458. 
16. Kumari, A.S., Pregnancy outcome in women with morbid obesity. Int J Gynaecol Obstet, 2001. 73(2): p. 101-7.

17. Du, M.-K., et al., Effects of pre-pregnancy body mass index and gestational weight gain on neonatal birth weight. Journal of Zhejiang University. Science. B, 2017. 18(3): p. 263-271.

18. Lei, Q., et al., Clustering of metabolic risk factors and adverse pregnancy outcomes: a prospective cohort study. Diabetes/Metabolism Research and Reviews, 2016. 32(8): p. 835-842.

19. Joy, S., et al., The Impact of Maternal Obesity on the Incidence of Adverse Pregnancy Outcomes in High-Risk Term Pregnancies. Am J Perinatol, 2009. 26(05): p. 345-349.

20. Liu, L., et al., Maternal body mass index and risk of neonatal adverse outcomes in China: a systematic review and meta-analysis. BMC Pregnancy and Childbirth, 2019. 19(1): p. 105.

21. Liu, P., et al., Association between perinatal outcomes and maternal pre-pregnancy body mass index. Obesity reviews : an official journal of the International Association for the Study of Obesity, 2016. 17.

22. Vinturache, A.E., et al., Perinatal outcomes of maternal overweight and obesity in term infants: a population-based cohort study in Canada. Scientific reports, 2015. 5: p. 9334-9334.

23. Zutshi, A., et al., Implications of Early Pregnancy Obesity on Maternal, Fetal and Neonatal Health: Retrospective cohort study from Oman. Sultan Qaboos University medical journal, 2018. 18(1): p. e47-e53.

24. Reynolds, R.M., et al., Maternal obesity during pregnancy and premature mortality from cardiovascular event in adult offspring: follow-up of 1323275 person years. Bmj, 2013. 347: p. f4539.

25. Mingrone, G., et al., Influence of maternal obesity on insulin sensitivity and secretion in offspring. Diabetes Care, 2008. 31(9): p. 1872-6.

26. Knight-Agarwal, C.R., et al., Association of BMI and interpregnancy BMI change with birth outcomes in an Australian obstetric population: a retrospective cohort study. BMJ Open, 2016. 6(5): p. e010667.

27. Bilal, P.I., C.K.Y. Chan, and S.M. Somerset, Acculturation and Perceived Ethnic Discrimination Predict Elevated Blood Glucose Level in Sub-Saharan African Immigrants in Australia. J Immigr Minor Health, 2020. 22(4): p. 771-777.

28. Pereira, C.A., N. Larder, and S. Somerset, Food acquisition habits in a group of African refugees recently settled in Australia. Health Place, 2010. 16(5): p. 934-41.

29. Holmboe-Ottesen, G. and M. Wandel, Changes in dietary habits after migration and consequences for health: a focus on South Asians in Europe. Food \& nutrition research, 2012. 56: p. 10.3402/fnr.v56i0.18891.

30. Jani, R., K.M. Mallan, and L. Daniels, Association between Australian-Indian mothers' controlling feeding practices and children's appetite traits. Appetite, 2015. 84: p. 188-195.

31. Dobbins, T.A., et al., Australian national birthweight percentiles by sex and gestational age, 1998-2007. Medical Journal of Australia, 2013. 198(4): p. 189-189.

32. Li, Z., et al., Birthweight percentiles by gestational age for births following assisted reproductive technology in Australia and New Zealand, $2002-2010$. Human Reproduction, 2014. 29(8): p. 1787-1800.

33. Jani, R., et al., The Association Between Pre-Pregnancy Body Mass Index, Perinatal Depression and Maternal Vitamin D Status: Findings from an Australian Cohort Study. International journal of women's health, 2020. 12: p. 213-219.

34. Snowden, J.M., et al., The Impact of maternal obesity and race/ethnicity on perinatal outcomes: Independent and joint effects. Obesity, 2016. 24(7): $\mathrm{p}$. 1590-1598. 